\title{
Comparative study of isoflurane and ketamine anesthetics in Rabbits
}

\author{
Muhammad Ali Khan ${ }^{1}$, Ahmed Nawaz Tunio ${ }^{1}$, Abrar Ahmad ${ }^{2 *}$, Allah \\ Baksh Kachiwal ${ }^{3}$, Mool Chand Malhi ${ }^{3}$, Asad Khan ${ }^{2}$, Muhammad \\ Qasim $^{4}$, Arsalan Said ${ }^{2}$, Rahat-Ullah Khan ${ }^{5}$ and Mirwaise Khan ${ }^{6}$
}

1. Department of Surgery \& Obstetrics, Faculty of Animal Husbandry \& Veterinary Sciences, Sindh Agriculture University Tandojam-Pakistan

2. Department of Biosciences, Faculty of Veterinary \& Animal Sciences, Gomal University D.I. Khan-Pakistan

3. Department of Physiology \& Biochemistry, Faculty of Animal Husbandry \& Veterinary Sciences, Sindh Agriculture University Tandojam-Pakistan

4. Department of Parasitology, University of Veterinary \& Animal Sciences, Lahore-Pakistan

5. Institute of Microbiology, Gomal University D.I. Khan-Pakistan

6. Department of Clinical Medicine and Surgery, Faculty of Veterinary \& Animal Sciences, Gomal University D.I.

Khan-Pakistan

*Corresponding author's email: abrarvet107@gu.edu.pk

Citation

Muhammad Ali Khan, Ahmed Nawaz Tunio, Abrar Ahmad, Allah Baksh Kachiwal, Mool Chand Malhi, Asad Khan, Muhammad Qasim, Arsalan Said, Rahat-Ullah Khan and Mirwaise Khan. Comparative study of isoflurane and ketamine anesthetics in Rabbits. Pure and Applied Biology. Vol. 8, Issue 2, pp1385-1397.

http://dx.doi.org/10.19045/bspab.2019.80079

Received: 07/01/2019 Revised: 22/04/2019

Accepted: 26/04/2019

Online First: 02/05/2019

\section{Abstract}

The current study was aimed to establish best anesthetic protocols in rabbit, for this purpose six clinically healthy male New Zealand White rabbits weighing $2 \pm 00 \mathrm{~kg}$ were procured. All rabbits received two anesthetic treatments with one week interval. Treatment: A Ketamine $35 \mathrm{mg} / \mathrm{kg} \mathrm{IM}$ and Xylazine $5 \mathrm{mg} / \mathrm{kg}$ IM, Treatment B: Isoflurane (3 to $5 \%$ ) through facemask followed by Xylazine $5 \mathrm{mg} / \mathrm{kg} \mathrm{IM}$. Physiological parameters such as, heart rate, rectal temperature and respiratory rate were recorded before and after administration of anesthetics in all animals. Heart rate of all animals significantly $(\mathrm{P}<0.05)$ decreased with Xylazine and increased significantly $(\mathrm{P}<0.05)$ with Ketamine at 10 minutes and at 15 minutes with Isoflurane after induction of anesthesia. Respiratory rates were significantly decreased $(\mathrm{P}<0.05)$ at 15 and 10 minutes in Group A and B respectively. No significant difference was observed in rectal temperature in both treatment groups. The onset and degree of sedation was non-significant $(\mathrm{P}>0.05)$ in both treatment groups. Induction of anesthesia was same in both treatment groups and was non-significant at $(\mathrm{P}>0.05)$. Isoflurane showed a significantly $(\mathrm{P}<0.05)$ longer duration of Anesthesia compared to Ketamine. Relaxation of muscle increased significantly $(\mathrm{P}<0.05)$ with Isoflurane compared to ketamine. Duration of analgesia and recovery from anesthesia was also found significant $(\mathrm{P}<0.05)$ with Isoflurane compared with ketamine. It was concluded that Isoflurane is the safe anesthetic agent for rabbits as an alternative of ketamine for longer duration of anesthesia and smooth recovery with enhanced analgesia.

Keywords: Anesthesia; Isoflurane; Ketamine; Physiology; Rabbits

Introduction

The domestic rabbit (Oryctolagus cunicuclus) being docile is extensively used as an animal model for a number of medico- veterinary practices such as teaching and research purpose, and especially in the fields where laboratory techniques and in vivo studies for various pharmacological and 
toxicological effects are sought [1]. It is also reared for fur industry, for meat and kept as a companion animal in several countries. Anesthesia is the reversible loss of consciousness, which is induced with many types of anesthetic agents. It is the art and choice of inducing analgesia, immobility and relaxed muscle tone for safe and smooth surgery and ease to the operator [2]. A variety of surgical procedures are performed using anesthesia including sedation for blood collection, as rabbits became stressed while handling therefore sedation is required, Intravenous cannula placement, radiography [3], and other operative measures such as neutering, gastrotomy, cystotomy and fracture fixation [4]. Administering anesthetics in rabbits is considered safe, when all safety measures are taken which includes careful check-up of the rabbit, the correct anesthetics and recommended dosage regimen and its maintenance and proper usage of equipment [5]. In addition pre anesthetics are also given along with general anesthetics, usually xylazine hydrochloride is administered in most animals which decrease the stress, calm the animal and reduce the total dose of general anesthetics [6]. Various anesthetic agents are used to perform surgical procedures on laboratory animals including rabbit such as Ketamine, Propofol, Sevoflurane and Isoflurane among others.

Isoflurane is an inhalant anesthetic that produce general anesthesia in all species including reptile, birds and zoo animals. They produce comparatively rapid induction and safe recovery from anesthesia [7]. Isoflurane causes rapid heart rate and increased myocardial contraction in contrast to other inhalant anesthetics which depress blood pressure due to decrease cardiac output [8]. It causes bradycardia in rabbits and it becomes violent while inhaling Isoflurane and causes deep and prolong breathing [9].

Ketamine is a dissociative anesthetic agent which causes poor muscle relaxation and increased sympathomimetic tone. Tonic clonic cramps and convulsion are the major signs of the side effects encountered during its usage, however along with a potent sedative it shows an excellent relaxed muscle tone [10].

As expected several species need distinct concentrations of anesthetics. However, the anesthetic effect with volatile nature differs very slightly among species with same concentration as those needed for human beings, rodents, amphibians and insects [11]. So far little work has been conducted to compare the physiological and anesthetic effect of ketamine and Isoflurane anesthetics in rabbits. The present study was conducted to establish improved protocol of anesthetics administration techniques in rabbits and for animal welfare generally, to investigate the effects intervening anesthesia and subsequent physiological effects of Isoflurane and ketamine in rabbits undergoing general anesthesia. The present study would help in the safer use of anesthetics in lab and large animals as well.

\section{Materials and methods Model animals}

For the current study six clinically healthy male rabbits (White New Zealand) were purchased from the local market of Hyderabad Sindh, Pakistan weighing approximately $2 \pm 00 \mathrm{Kg}$ and aged between 46 months.

\section{Management of experimental animals}

The rabbits were allowed for 15 days acclimatization period in the experimental station of the Department of Surgery \& Obstetrics, Faculty of Animal Husbandry and Veterinary Sciences, Sindh Agriculture University Tandojam. The rabbits were fed on seasonal fresh grass, Fresh vegetables and water ad libitum.

\section{Experimental design}

The current study was performed during two consecutive weeks such as at $1^{\text {st }}$ week and $2^{\text {nd }}$. Two different anesthetic treatments were administered using randomized cross over 
design to every rabbit. Each rabbit received both anesthetic treatments with one week interval. Treatment A include Ketamine at dose rate of $35 \mathrm{mg} / \mathrm{kg}+$ Xylazine $5 \mathrm{mg} / \mathrm{kg} \mathrm{IM}$ and Treatment B contain Isoflurane 3 to $5 \%$ using face mask + Xylazine $5 \mathrm{mg} / \mathrm{kg}$ IM (Table 1).

\section{Ethical approval}

The study plan was discussed by the Board of study of DAS (Directorate of Advance Studies) and was approved by the ethical committee.

Table 1. Experimental Design of rabbits used in the study

\begin{tabular}{|c|c|c|}
\hline Animals & $\mathbf{1}^{\text {st }}$ week & $\mathbf{2}^{\text {nd }}$ week \\
\hline Rabbit 1 & A & B \\
\hline Rabbit 2 & B & A \\
\hline Rabbit 3 & A & B \\
\hline Rabbit 4 & B & B \\
\hline Rabbit 5 & A & A \\
\hline Rabbit 6 & B & \\
\hline
\end{tabular}

$\mathrm{A}=$ Ketamine $(35 \mathrm{mg} / \mathrm{kg}) \mathrm{IM}, \mathrm{B}=$ Isoflurane (3-5\%) with facemask

\section{Evaluation of sedative and anesthetic effects}

The following physiological parameters were recorded in all rabbits. Rectal temperature, heart rate and respiratory rate were recorded along with other reflexes such as, palpebral and corneal reflexes, ear pinch, toe pinch, jaw tone during the anesthetics protocol and its side effects were monitored for Isoflurane and ketamine during anesthesia in Rabbits. All parameters were recorded after every 5 minutes interval.

\section{Heart Rate}

The heart rate (beats/minutes) was recorded before administration of any drug as control, at five minutes after administration of Xylazine and then at every 5 minutes up to 60 minutes of anesthesia.

\section{Respiratory rate (Per/Min)}

Respiration rate (breaths/minutes) was recorded before as a control, at five minutes after Xylazine and then at every 5 minutes up to 60 minutes of anesthesia.

\section{Rectal Temperature $\left({ }^{0} \mathbf{F}\right)$}

Body temperature was determined before administration of any drug as a control, at five minutes after Xylazine and then after every 5 minutes up to 60 minutes.

\section{The stages of sedation and anesthetic influence}

The stage, time period of sedation, anesthesia duration, standing time, set in recumbency and time period of recumbency in each rabbit along with treatment were noticed. Analgesic effect was examined by no response using deep needle pricking. Induction and presence/absence of apnea was noted in each rabbit.

\section{Statistical analysis data}

The obtained data were subjected to single way analysis of variance (ANOVA) and analyzed data along with Tukey -Karmer Multiple Comparison test.

\section{Results}

The data collected on the anesthetic effect of Isoflurane and Ketamine along with the preanesthetic Xylazine hydrochloride in a rabbit model are presented.

\section{Heart rate (beats/minute)}

The mean control values of heart rate of rabbits with treatment $\mathrm{A}$ and $\mathrm{B}$ were $209 \pm 14.89$ and $253 \pm 16.27$ respectively.

The heart rate (HR) of all animals after xylazine administration was decreased significantly $(\mathrm{P}<0.05)$. But after induction of anesthetic agent the heart rate started increasing significantly $(\mathrm{P}<0.05)$ from the 
pre-anesthetic value in all groups. Comparison of the two treatment showed that the heart rate after 5 minutes of induction increased $(\mathrm{P}<0.05)$ with treatment $\mathrm{A}$ at 10 minutes ( 150 beats/minute) and at 15 minutes (162.66 beats/minute) with treatment B after induction of anesthesia (Table 2, Figure 1).

Table 2. Mean values of heart rate (per minutes) after administration of drugs in Rabbits

\begin{tabular}{|c|c|c|c|c|}
\hline $\begin{array}{c}\text { Time } \\
\text { (Minutes) }\end{array}$ & \multicolumn{2}{c|}{$\begin{array}{c}\text { A } \\
\text { Xylazine+Ketamine }\end{array}$} & \multicolumn{2}{c|}{ Xylazine+Isoflurane } \\
\hline & Mean & \pm SEM & Mean & \pm SEM \\
\hline Control & $* 209$ & \pm 14.89 & $* 253$ & \pm 16.27 \\
\hline 5 P & 113 & \pm 0.51 & 164 & \pm 10.11 \\
\hline 5 I & 165 & \pm 7.54 & 158 & \pm 5.05 \\
\hline 10 & $150^{*}$ & \pm 6.00 & 163 & \pm 3.60 \\
\hline 15 & 154 & \pm 4.81 & $162.66^{*}$ & \pm 4.7796 \\
\hline 20 & 156.66 & \pm 5.40 & 163 & \pm 3.60 \\
\hline 25 & 157 & \pm 5.88 & 161.33 & \pm 2.71 \\
\hline 30 & 157 & \pm 5.45 & 168.33 & \pm 3.07 \\
\hline 35 & 153 & \pm 7.54 & 168.33 & \pm 3.07 \\
\hline 40 & 153 & \pm 7.54 & 168.33 & \pm 4.63 \\
\hline 45 & 157 & \pm 11.26 & 173.33 & \pm 2.47 \\
\hline 50 & 160 & \pm 6.69 & 173.16 & \pm 1.64 \\
\hline 55 & 160 & \pm 6.69 & 174.16 & \pm 3.51 \\
\hline 60 & 170.5 & \pm 5.72 & 180 & \pm 5.73 \\
\hline 65 & 170.40 & \pm 2.85 & 190.83 & \pm 2.71 \\
\hline 70 & 174.80 & \pm 2.41 & 198.33 & \pm 4.59 \\
\hline
\end{tabular}

*Indicate significantly lowest value in corresponding column as compared to control, $\mathbf{P}=$ Pre anesthetics, $\mathbf{I}=$ Induction of anesthesia

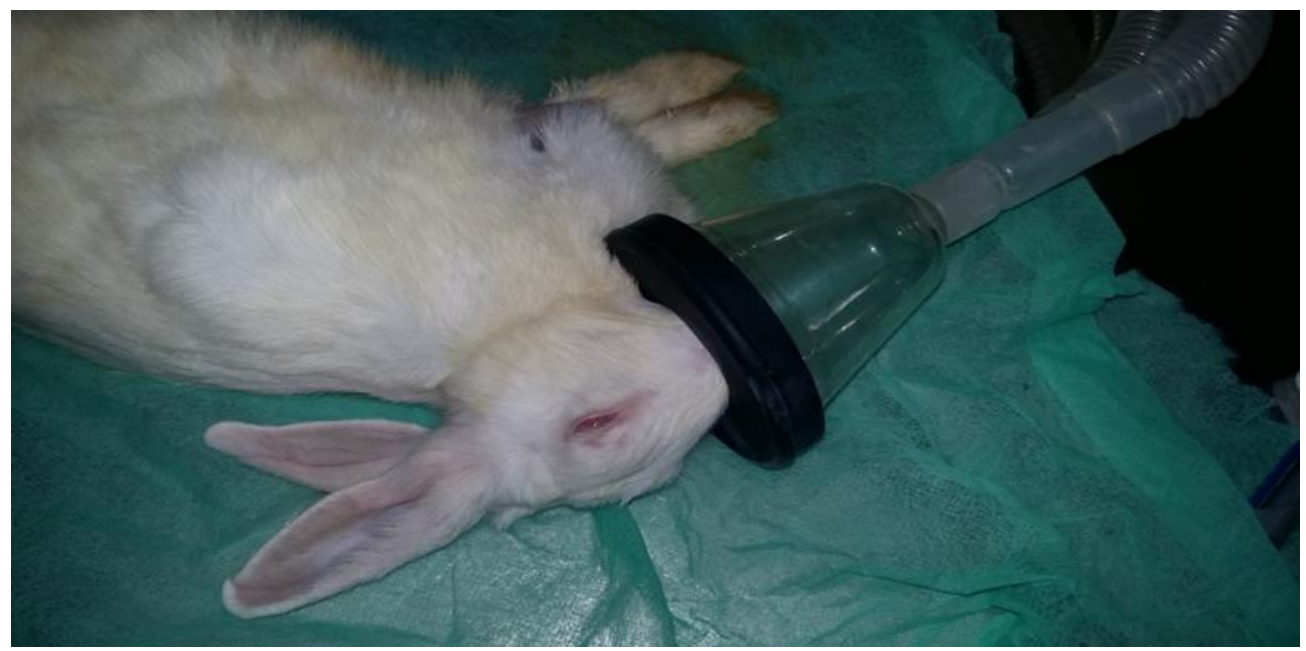

Figure 1. Assessment of heart rate (per minute) after administration of Isoflurane in rabbits. Monitoring of the respiratory rates

The mean control respiratory rate of rabbits with treatments $\mathrm{A}, \mathrm{B}$ were $145 \pm 12.81$ and $156 \pm 7.42$ breaths per minute respectively.
These values were significantly $(\mathrm{P}<0.05)$ different from each other. 
The reduction of respiratory rate $(\mathrm{P}<0.05)$ was seen after the administration of xylazine. After 5 minutes of induction of anesthesia respiratory rate decreased significantly $(\mathrm{P}<0.05)$ from control values with all treatments. The maximum decrease was recorded at 15 minutes with treatment $\mathrm{A}$, at 10 minutes with treatment $\mathrm{B}$. It then started to increase and reach the base line values after 70 minutes in both treatment groups (A and B).

Comparison of the two treatments showed that there was a significant difference $(\mathrm{P}<0.05)$ in respiratory rates during anesthesia (Table 3).

Table 3. Mean values of respiratory rate (Per minute) after administration of drugs in rabbits

\begin{tabular}{|c|c|c|c|c|}
\hline $\begin{array}{c}\text { Time } \\
\text { (Minutes) }\end{array}$ & \multicolumn{2}{|c|}{ A } & \multicolumn{2}{c|}{ B } \\
\hline (Minutes) & Mean & \pm SEM & Mean + Isoflurane \\
\hline Control & $* 145$ & \pm 12.81 & $* 156$ & \pm SEM \\
\hline 5 P & 4.33 & \pm 10.08 & 61.66 & \pm 7.42 \\
\hline 5 I & 46.66 & \pm 6.21 & 45 & \pm 9.35 \\
\hline 10 & 44.33 & \pm 6.66 & $43 *$ & \pm 2.56 \\
\hline 15 & $37 *$ & \pm 2.86 & 40 & \pm 2.86 \\
\hline 20 & 39.66 & \pm 5.69 & 43 & \pm 2.00 \\
\hline 25 & 39.66 & \pm 5.69 & 43 & \pm 2.86 \\
\hline 30 & 41.66 & \pm 5.12 & 43.5 & \pm 2.86 \\
\hline 35 & 41.66 & \pm 5.12 & 47.5 & \pm 3.05 \\
\hline 40 & 41.66 & \pm 5.23 & 50.33 & \pm 2.65 \\
\hline 45 & 42.66 & \pm 5.23 & 58.5 & \pm 2.01 \\
\hline 50 & 44.66 & \pm 4.86 & 71.83 & $\pm 3,35$ \\
\hline 55 & 44.66 & \pm 4.86 & 78.33 & \pm 2.65 \\
\hline 60 & 51.66 & \pm 5.61 & 84 & \pm 5.25 \\
\hline 65 & 55.5 & \pm 10.50 & 111 & \pm 3.33 \\
\hline 70 & 62.5 & \pm 13.25 & 134.16 & \pm 3.51 \\
\hline
\end{tabular}

*Indicate significantly lowest value in corresponding column as compared to control. $\mathrm{P}=$ Pre anesthetics, $\mathrm{I}=\mathrm{Induction}$ of anesthesia

\section{Rectal temperature: $\left({ }^{0} \mathbf{F}\right)$}

The mean control temperature of rabbits with both treatment $\mathrm{A}$ and $\mathrm{B}$ were $103^{\circ} \mathrm{F}$. There was no prominent elevation in the values of temperature between the two groups with the administration of pre anesthetic Xylazine hydrochloride.

The temperature was not decreased after administering Isoflurane and Ketamine i.e.
Treatment A and B compared to the values of control group. The body temperature was remained constant throughout the period of anesthesia. Comparison of the two treatments showed that there was no any significant difference in the body temperature during anesthesia and recovery period (Table 4). 
Table 4. Mean values of temperature ( $\left.{ }^{0} \mathrm{~F}\right)$ after administration of drugs in Rabbits

\begin{tabular}{|c|c|c|c|c|}
\hline \multirow{2}{*}{ Time } & \multicolumn{2}{|c|}{ A } & \multicolumn{2}{c|}{ B } \\
& \multicolumn{2}{|c|}{ Xylazine ${ }_{+}$Ketamine } & \multicolumn{2}{c|}{ Xylazine + Isoflurane } \\
\hline Minutes & Mean & \pm SEM & Mean & \pm SEM \\
\hline Control & 103 & \pm 0.00 & 103.00 & \pm 0.00 \\
\hline 5 P & 103 & \pm 0.00 & 103.00 & \pm 0.00 \\
\hline 5 I & 103 & \pm 0.00 & 103.00 & \pm 0.00 \\
\hline 10 & 103 & \pm 0.00 & 103.00 & \pm 0.00 \\
\hline 15 & 103 & \pm 0.00 & 103.00 & \pm 0.00 \\
\hline 20 & 102.83 & \pm 0.16 & 103.00 & \pm 0.00 \\
\hline 25 & 102.80 & \pm 0.38 & 103.00 & \pm 0.00 \\
\hline 30 & 102.97 & \pm 0.23 & 103.00 & \pm 0.00 \\
\hline 35 & 102.97 & \pm 0.23 & 103.00 & \pm 0.00 \\
\hline 40 & 102.97 & \pm 0.23 & 103.00 & \pm 0.00 \\
\hline 45 & 102.83 & \pm 0.16 & 103.00 & \pm 0.00 \\
\hline 50 & 102.83 & \pm 0.16 & 103.00 & \pm 0.00 \\
\hline 55 & 102.83 & \pm 0.16 & 103.00 & \pm 0.00 \\
\hline 60 & 102.50 & \pm 0.50 & 103.00 & \pm 0.00 \\
\hline 65 & 102.5 & \pm 0.50 & 103.00 & \pm 0.00 \\
\hline 70 & 103 & \pm 0.00 & 103.00 & \pm 0.00 \\
\hline
\end{tabular}

$\mathbf{P}=$ Pre anesthetics, $\mathbf{I}=$ Induction of anesthesia

Onset and degree of sedation

Xylazine produced mild degree of sedation within $1.75 \pm 0.25$ minutes with both treatments A and B. There was no recumbency observed in any animal with all treatments (Figure 2). Xylazine produce mild degree of sedation $(+)$ with all treatments having a non-significant difference $(\mathrm{P}>0.05)$ (Table 5).

Table 5. Mean values for scoring of onset of sedation (minutes) and degree of sedation after administration of xylazine in rabbits

\begin{tabular}{|c|c|c|c|c|}
\hline Animal No & \multicolumn{2}{|c|}{ Onset of sedation } & \multicolumn{2}{c|}{ Degree of sedation } \\
\hline & A & B & A & B \\
\cline { 2 - 5 } & Xylazine & Xylazine & Xylazine & Xylazine \\
\hline 1 & 2 & 1 & 1 & 1 \\
\hline 2 & 1 & 2.5 & 1 & 1 \\
\hline 3 & 2 & 2 & 1 & 1 \\
\hline 4 & 2.5 & 1 & 1 & 1 \\
\hline 5 & 2 & 2 & 1 & 1 \\
\hline 6 & 1 & 2 & 1 & $1.00 \pm 00$ \\
\hline Mean \pm S.E & $1.75 \pm 0.25$ & $1.75 \pm 0.25$ & $1.00 \pm 00$ & \\
\hline
\end{tabular}

*Indicate significant difference $(\mathrm{P}<0.05)$ between corresponding parameter, Scoring of degree of sedation

$0=$ zero degree sedation, $1=$ less sedation than normal and more than zero, $2=$ sedation degree is high then normal, $3=$ deep sedation 


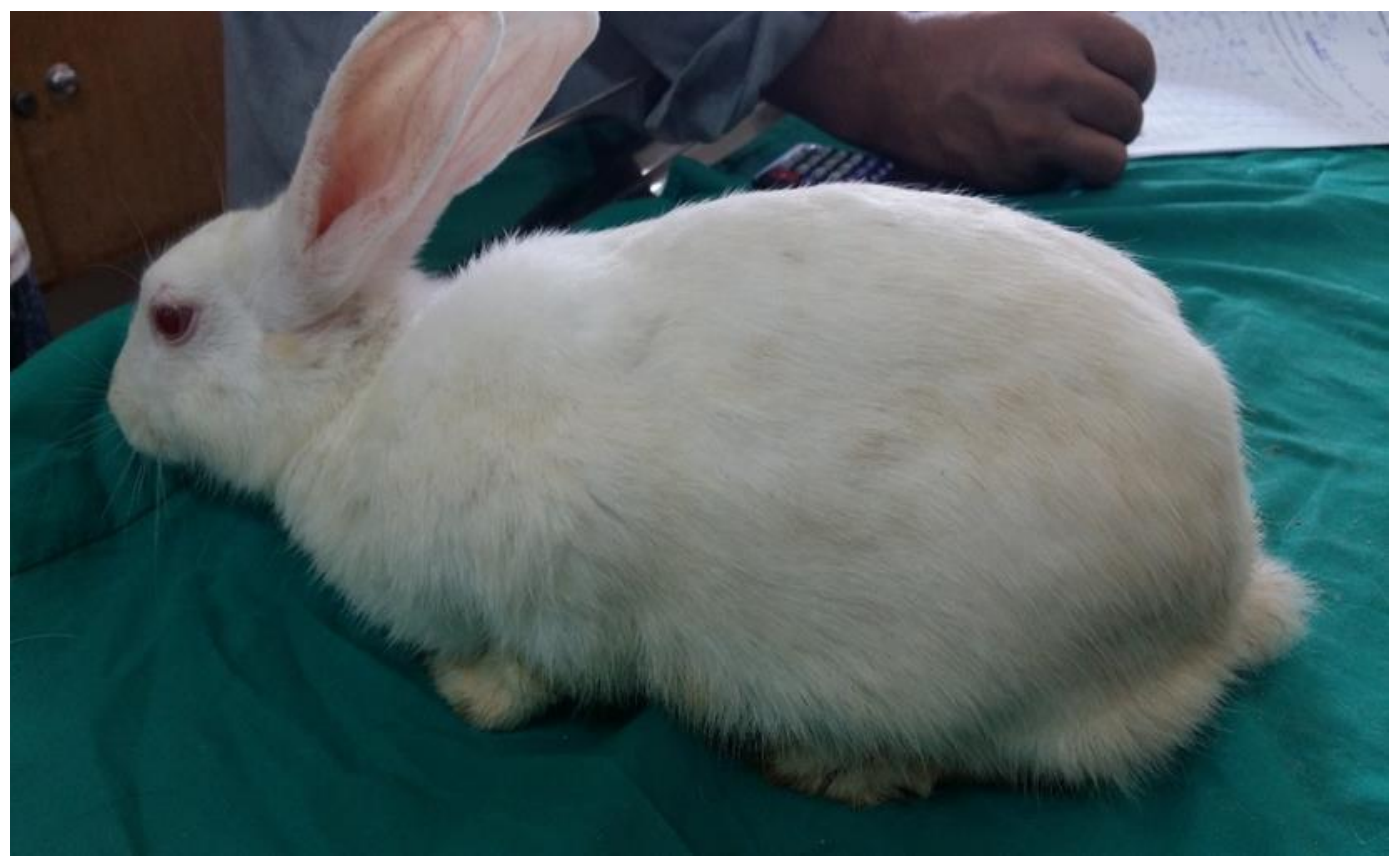

Figure 2. Onset of sedation (minutes) after administration of xylazine in rabbits

\section{Induction of anesthesia}

Induction of anesthesia after administration of different anesthetic drugs was $30 \pm 0.97$ and $30 \pm 0.97$ seconds with treatments A and B respectively. All animals were in standing position before induction of anesthesia
(Figure 3). The induction was equal, rapid and non-significant at $(\mathrm{P}>0.05)$ with both treatment groups. The induction was smooth, rapid and excitement free in all rabbits (Table $6)$.

Table 6. Induction of anesthesia (seconds) after administration of anesthetic agents in rabbits

\begin{tabular}{|c|c|c|}
\hline \multirow{2}{*}{ Animal No. } & \multicolumn{2}{|c|}{ Induction of anesthesia } \\
\cline { 2 - 3 } & Treatment \\
\hline 1 & 30 & B \\
\hline 2 & 28 & 30 \\
\hline 3 & 32 & 34 \\
\hline 4 & 26 & 32 \\
\hline 5 & 34 & 28 \\
\hline 6 & 30 & 30 \\
\hline Mean \pm S. E & $30 \pm 0.97$ & 26 \\
\hline
\end{tabular}

*Indicate significant difference $(\mathrm{p}<0.05)$ between corresponding parameter 


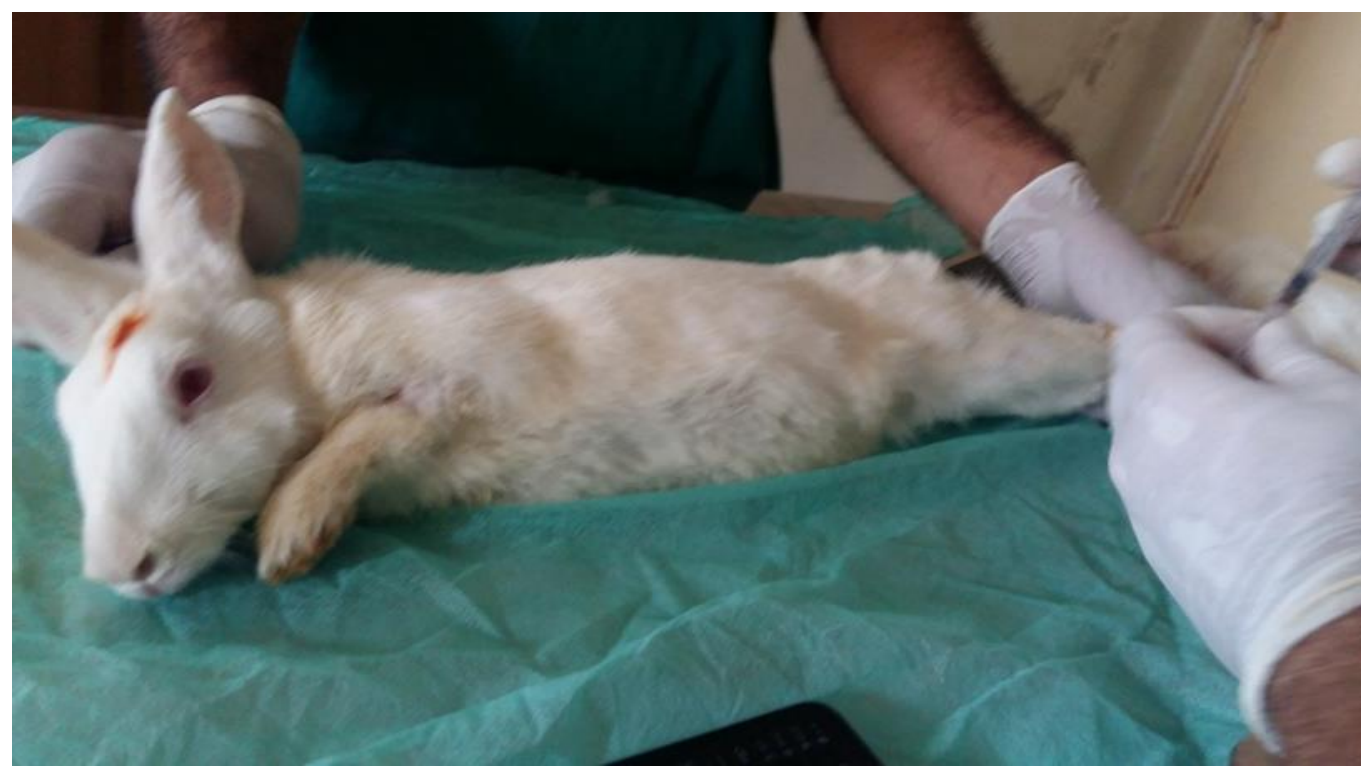

Figure 3. Induction of anesthesia (seconds) after administration of ketamine anesthetic agents in rabbits

\section{Duration and degree of anesthesia}

Table 7 represents the data regarding the duration and degree of anesthesia in rabbits administered inhalational and parenteral anesthetics. The mean duration of anesthesia after introduction were $52.64 \pm 7.76$ and $60.00 \pm 00$ minutes in Group $\mathrm{A}$ and $\mathrm{B}$ respectively. The duration of anesthesia of treatment $\mathrm{B}$ was significantly $(\mathrm{P}<0.05)$ longer as compared to treatment A. Surgical anesthesia (stage 3 - plane 3 ) were observed in all animals with treatments $\mathrm{A}$ and $\mathrm{B}$ (Table 7).

Table 7. Duration and degree of anesthesia (minutes) with anesthetic agents

\begin{tabular}{|c|c|c|c|c|}
\hline \multirow{3}{*}{ Animal No. } & \multicolumn{2}{|c|}{ Duration of anesthesia (minutes) } & \multicolumn{2}{|c|}{ Degree of anesthesia } \\
\hline & \multicolumn{4}{|c|}{ Treatment } \\
\hline & $\mathbf{A}$ & B & $\mathbf{A}$ & B \\
\hline 1 & 55 & 60 & 3 & 3 \\
\hline 2 & 50 & 60 & 3 & 3 \\
\hline 3 & 65 & 60 & 3 & 3 \\
\hline 4 & 55 & 60 & 3 & 3 \\
\hline 5 & 60 & 60 & 3 & 3 \\
\hline 6 & 55 & 60 & 3 & 3 \\
\hline Mean \pm S.E & $52.64 \pm 7.76$ & $* 60.00 \pm 00$ & $3.00 \pm 00$ & $3.00 \pm 00$ \\
\hline
\end{tabular}

*Indicate significant difference $(\mathrm{P}<0.05)$ between corresponding parameter

\section{Degree of muscle relaxation}

One of the major target of anesthetics is to achieve maximum muscle relaxation to smoothly proceed any sort of surgical intervention in majority of veterinary procedures. Table- 8 depicts the comparative degree of relaxed muscle tone in both treatment groups. Degree of muscles relaxation was found statistically significant $(\mathrm{P}<0.05)$ with treatment $\mathrm{B}$ as compared to treatment $\mathrm{A}$ after administering Isoflurane and Ketamine anesthetics respectively (Table 8). 
Table 8. Degree of muscle relaxation

\begin{tabular}{|c|c|}
\hline \multicolumn{2}{|c|}{ Degree of muscle relaxation } \\
\hline \multicolumn{2}{|c|}{ Treatment } \\
\hline $\mathbf{A}$ & B \\
\hline 2 & 3 \\
\hline 2 & 3 \\
\hline 2 & 3 \\
\hline 2 & 3 \\
\hline 2 & 3 \\
\hline 2 & 3 \\
\hline $2.00 \pm 00$ & $* 3.00 \pm 00$ \\
\hline
\end{tabular}

*Indicate significant difference $(\mathrm{P}<0.05)$ between corresponding parameters

Degree of muscle relaxation, 1= Mild, 2= Moderate 3= Deep

Duration of analgesia (minutes) and degree of analgesia

The mean duration of analgesia were $51.66 \pm 7.76$ and $60.00 \pm 00$ minutes after the administration of Ketamine (Group A) and Isoflurane (Group B) anesthetics treatment respectively. The statistical analysis with one way ANOVA revealed significantly longer $(\mathrm{P}<0.05)$ duration of analgesia with treatment $\mathrm{B}$ as compared to treatment A, However the degree of analgesia was found deeper (Grade-3) in both treatment groups i.e Treatment A and B (Table 9).

Table 9. Duration of analgesia (minutes) and degree of analgesia

\begin{tabular}{|c|c|c|c|c|}
\hline Animal no & \multicolumn{3}{|c|}{ Duration of analgesia (minutes) } & \multicolumn{3}{c|}{ Degree of analgesia } \\
\hline & \multicolumn{3}{|c|}{ Breatment } \\
\cline { 2 - 5 } & A & 60 & A & B \\
\hline 1 & 50 & 60 & 3 & 3 \\
\hline 2 & 45 & 60 & 3 & 3 \\
\hline 3 & 60 & 60 & 3 & 3 \\
\hline 4 & 50 & 60 & 3 & 3 \\
\hline 5 & 55 & 60 & 3 & $3.00 \pm 00$ \\
\hline 6 & 50 & $* 60.00 \pm 00$ & $3.00 \pm 00$ & \\
\hline Mean \pm S.E & $* 51.66 \pm 7.76$ & &
\end{tabular}

*Indicate significant difference $(\mathrm{P}<0.05)$ between corresponding parameters,

1= Mild 2= Moderate 3= Deep

\section{Recovery from anesthesia}

Recovery from anesthesia was almost safe and excitement free with both treatment groups i.e A and B. Complications such as cardiac arrest, respiratory arrest, respiratory depression, excitement, nerve paralysis and vomiting were absent during anesthesia and recovery period.

\section{Recovery period}

The data regarding the recovery phase from the anesthetic effect are displayed in (Table 10), the mean values for recovery time with treatment $\mathrm{A}$ and $\mathrm{B}$ was $19.66 \pm 2.45$ and $1.00 \pm 0.00$ minutes respectively. Duration of recovery with treatment B (Isoflurane) was shorter than treatment A (Ketamine), showing a statistically significant difference $(\mathrm{P}<0.05)$ between the two groups (Table 10). 
Table 10. Recovery period from anesthesia in rabbits

\begin{tabular}{|c|c|c|}
\hline \multirow{2}{*}{ Animal No. } & \multicolumn{2}{|c|}{ Recovery from anesthesia (minutes) } \\
\cline { 2 - 3 } & Treatment \\
\hline 1 & 14 & B \\
\hline 2 & 25 & 1 \\
\hline 3 & 20 & 1 \\
\hline 4 & 11 & 1 \\
\hline 5 & 26 & 1 \\
\hline 6 & 22 & 1 \\
\hline Mean \pm S. E & $* 19.66 \pm 2.45$ & $* 1.00 \pm 0.00$ \\
\hline
\end{tabular}

*Indicate significant difference $(\mathrm{p}<0.05)$ between corresponding parameter

\section{Other observations}

Besides recording of basic physiological parameters during the course of the study other observations such as, palpebral reflex, corneal, ear pinch, toe pinch and jaw tone were also observed in the animals ongoing anesthesia period in both treatment groups. Palpebral reflexes were present in treatment A but absent with treatment B, however all other reflexes were not seen in both groups (Table 11).

Table 11. Summary of other observations/reflexes distinguished during treatment A and B

\begin{tabular}{|c|c|c|}
\hline \multirow{2}{*}{ Reflexes } & \multicolumn{2}{|c|}{ Treatment } \\
\cline { 2 - 3 } & A & B \\
\hline Palpebral & Seen & Unseen \\
\hline Corneal & Unseen & Unseen \\
\hline Ear pinch & Unseen & Unseen \\
\hline Toe pinch & Unseen & Unseen \\
\hline Jaw tone & Unseen & Unseen \\
\hline
\end{tabular}

\section{Discussion}

Anesthesia and sedation methods are the most important elements to perform safe and painless surgical dealings. Effective anesthetic protocols are important for experimental animals as well as other clinical patients, not only for surgical procedures but also for safe handling and diagnostic procedures [12]. In this study two general anesthetic protocols were used and their anesthetic effects were compared using six rabbits receiving two treatments. No mortality was observed in any group during the experiment trial. All rabbits were kept under hygienic condition in the indoor patient ward of Department of Surgery and Obstetrics. Only one animal (Rabbit No 5) had shown hyperthermia on second day of
Ketamine anesthesia then it was treated with injection of Amoxicillin 0.5ml (I.M) and was found normal on next day. The increase in temperature in one rabbit in case of ketamine administration might be attributed to an idiosyncratic response towards ketamine.

General anesthetics are considered safer for rabbits, when all safety measure are adopted which are achieved on complete physical and routine examinations before any anesthesia. Intramuscular route is commonly used in rabbits and Intravenous is the best route for anesthesia via ear vein. However, inhalational anesthesia is also considered a safer protocol for induction in rabbits. Many safe and effective anesthetics have been studied in rabbit model. It is well known that inhalation anesthesia is safer as compared to 
injectable anesthesia in rabbits. But due to limited facility of gas anesthesia and the lack of equipment in hospitals they are neglected. In this study one injectable and one inhalational anesthetic were administered to rabbits with xylazine as pre-anesthetic as followed by [13].

Heart rate of rabbits administered with Isoflurane (3-5 \%) were significantly increased as compared to Ketamine $(35 \mathrm{mg} / \mathrm{kg})$. These findings are analogous to another study who observed that heart rate increased continuously in anesthetic with ketamine 200 which was seen in first ten minutes [14]. The effect of heart rate and minimal alveolar pressure co-relation are much stable in ketamine 100 and ketamine 200 [15]. In another study $2 \mathrm{mg} / \mathrm{kg}$ of ketamine showed non-significant alteration in parameters relating hemodynamics [16]. Ketamine and xylazine combination used I/V at $35 \mathrm{mg} / \mathrm{kg}(\mathrm{K})$ and $5 \mathrm{mg} / \mathrm{kg}(\mathrm{X})$ showed a significant increase in vessel diameter and considerably decreased heart rate, although mean arterial blood pressure primarily increased in rabbits [17].

Respiratory rate of rabbits administered were significantly higher in Isoflurane (3-5\%) as compared to Ketamine $(35 \mathrm{mg} / \mathrm{kg}$ ) our results did not correspond with [18], who reported that ketamine causes depression in respiration. The increase in respiratory rate with Isoflurane might be due to its formulation for inhalational route and large surface area for absorption [19]. When ketamine infusion administered it shows little bit respiratory depression along with hypercapnia. Hypoxia occurs as reported by $[20,21]$, in the present study there were no signs of hypoxia that might be due to the availability of Oxygen during anesthesia. In another study a significant decrease in respiratory rate found between $5-30$ minutes reading time [16], this decrease respiratory rate may be due to mixing more than one anesthetic drugs which causes inhibition of brain stem respiratory centers and alteration in blood flow [22]. The present fact is also supported by [23]. All anesthetic methods used along with the administration of oxygen in rabbit which shows alteration of lungs and ventilation process is depressed due to ketamine [24].

Rectal temperature was not affected significantly in both groups using Isoflurane and Ketamine. The present findings were in agreement with [16]. Our results are in line with [25] who showed non-significant difference $(\mathrm{P}>0.05)$ in rectal temperature in all groups when used different anesthetic protocol in rabbit, and disagreement with [26] who showed increase rectal temperature, however they used xylazine alone.

In current study, xylazine produced slight degree of sedation (+). This is parallel with the study [16] they observed significant deep analgesia, and muscle relaxation between 2060 minutes with xylazine. Early induction of anesthesia at $(30 \pm 0.97$ second $)$ in rabbits were observed with the administration of Isoflurane and, while late induction of anesthesia at Ketamine in rabbits were examined. [15, 18, 27]. Ketamine effects the central nervous system in human and increase peripheral vascular resistance [28]. Duration of anesthesia (60.00 \pm .00 minutes) were observed longer after administration of Isoflurane in rabbits while shorter for Ketamine (52.46 \pm 7.76 minutes). These findings are in agreement with [16].

Early recovery from anesthesia at (1.00 minutes) was noted in rabbits administered with Isoflurane, while medium recovery from anesthesia (16.6 minutes) was examined in rabbits administered with ketamine. These results are in line with [29] and in contrast with [30]. They found recovery time with ketamine at 120 minutes. This variation might be due to formulation difference of ketamine, handling and administration protocols. Furthermore sex, health, age, strain and nutritional status of individual 
animal also contribute to different responses. The use of Ketamine and Isoflurane shows the rapid response for maintenance in present study. The recovery time in all animals of both groups was smooth, with no signs of pain at the site of injection.

\section{Conclusion}

It is concluded that Isoflurane was the safest anesthesia in rabbits for all surgical procedure as it has smooth induction, good and faster recovery and no complications. Ketamine have the disadvantages like tachycardia and longer duration of recovery.

\section{Authors' contributions}

Conceived and designed the experiments: AN Tunio \& AB Kachiwal, Performed the experiments: MA Khan, Analyzed the data: MC Malhi \& A Said, Contributed materials/ analysis/ tools: A Khan, M Qasim, RU Khan \& M Khan, Wrote the paper: A Ahmad.

\section{Acknowledgement}

The Authors are grateful to the Department of Surgery \& Obstetrics, Faculty of Animal Husbandry \& Veterinary Sciences, Sindh Agriculture University Tandojam for providing facilities \& technical support for the research work.

\section{References}

1. Hristov H, Kostov D \& Vladova D (2006). Topographical anatomy of some abdominal organs in rabbits. Tra J Sci 4(3): 7-10.

2. Paul A \& Flecknel K (2009). Anesthesia and its monitoring in rabbits. Lab Animal Anaesth 3: 79-108.

3. Hubrecht RC \& Kirkwood J (2010). The UFAW handbook on the care and management of laboratory and other research animals. John Wiley \& Sons 399-417.

4. Borkowski R \& Karas AZ (1999). Sedation and anesthesia of pet Rabbit. Clinical tech in small Ani Prac 14(1): 44-49.
5. Praag (2016). Anesthesia and its monitoring in rabbits. Email:info@medirabbit.com.

6. Hall LW, Clarke KW, Trim CM \& Saunder WB (2003). Vet Anesthesia 10: 83-87.

7. Muir WW, Hubbenll JE, Skarda RT \& Bednarski RM (2000). Inhalant anesthetics A hand book of Veterinary anesthesia, pp 3: 19-23

8. Steffey EP, Kelly AB, Hodgson DS, Grandy JL, Woliner MJ \& Willits N (1990). Effect of body posture on cardiopulmonary function in horses during five hours of constant-dose halotane anesthesia. Anim J Vet Res 51: 6-11.

9. Flecknell PA \& Mitchell M (1984). Midazolam and fentanyl-fluanisone: assessment of anesthetic effects in laboratory rodents and rabbits. Lab. Anim. 18:143-146.

10. Short C, Baltimore E, Williams H \& Wilkins. 1987. Dissociative Anesthesia. Princ Prac Vet Anes 158: 115-117.

11. Liu J, Hu ZY, Ye QQ \& Dai SH (2009). Development of three Drosophila melanogaster strains with different sensitivity to volatile anesthetics. Chi Med J 122: 561-565.

12. Durrani UF, Ashraf $M$ \& Khalid A (2005). Comparative efficacy of Detomidine and Detomidine - ketamine cocktail in quails. Pak Vet J 25(4): 197199.

13. Popilskis SJ, Ozz MC \& Gorman $\mathrm{P}$ (1991). Comparison of xylazine with Tiletamine-Zolazepam (Telazol) and xylazine-ketamine anesthesia in rabbits. Lab Anim Sci 41: 51-53.

14. Carregaro (2016). Propofol application in veterinary sedation and anesthesia. Lab Animal Pract 17: 4-5.

15. Lerche P, Nolan AM \& Reid J (2000). Comparative study of propofol and 
ketamine for the induction of anesthesia in dogs. Vet Rec 146: 571-574.

16. Ahmed A, Al Qarwa AA \& Musa AM. (2014). Aphradynamic study of Propofol and ketamine infusion in ponies undergoing surgery. Res Vet Sci 64: 15964.

17. Xu Q, Ming Z, Dart AM, Du XJ (2007). Optimizing dosage of ketamine and xylazine in murine

echocardiography. Clin Exp Pharma Physio 34: 499-507.

18. Glowaski MM \& Wetmore A (1999). Propofol application in Veterinary sedation and anesthesia. Clin Tech Small Anim Pract 14: 1-9.

19. Wolfensberger BR \& Larenza MP (2007). Balanced anesthesia in the equine. Clin Tech Equi Pract 6: 104110.

20. Peeters ME, Gil D \& Teske E (1988). Four methods for general anaesthesia in the rabbit a comparative study. Lab. Ani. 22: 355-360.

21. Hellebrekers LJ, De Boer EJ \& Van Zuylen MA (1997). A comparison between medetomidine, ketamine and medetomidine-propofol anesthesia in rabbits. Lab Anim 31: 58-69.

22. Hutton S, Avramov MN, Shadrick M, Witt TR \& Ivankovich AD (2002). The use of a ketamine-propofol combination during monitored anesthesia care. Anesth Analg 90: 858-62.

23. Adetunji A, Ajadi RA \& Adewoye CO (2002). Total intravenous Anaesthesia with propofol: repeat bolus versus continuous Propofol infusion technique in xylazine-premedicate dogs. Israel $J$ Vet Med 52: 139.

24. Orr HE, Roughan JV \& Flecknell PA (2005). Assessment of ketamine and medetomidine anaesthesia in the domestic rabbit. Vet Anaesth Analg 32: 271-275.

25. Jasim D. Hankle J \& Brill J (2011). Comparative study of different intramuscular anesthesia combination in rabbit. Vet Anesth Analg 34: 274-280.

26. Egwn JV, Booth NH, Hatch RC \& Wallner B. (2011). Antagonism of xylazine sedation by 4- aminopyridine and yohimbine in cattle. Am J Vet Res 43: 2165-2169.

27. Baumgartner $\mathrm{C}$, Bollerhey $\mathrm{M} \&$ Henke $\mathrm{J}$ (2007). Effects of Propofol on ultrasonic indicators of hemodynamic function in rabbits. Vet Anaesth Analg 35: 100-112.

28. Tweed WA, Minuck MS \& Mymin D (1972). Circulatory response to ketamine anesthesia. Anesthesiol 37: 613-619.

29. Allweiler A, Knobloch M \& Portier G (2010). Additive interaction between Propofol and ketamine when used for anesthesia induction in female patient. Anaesthesiol 85: 751-759.

30. Hedenqvist P, Roughan JV, Orr HE \& Antunes LM (2001). Assessment of ketamine/medetomidine anaesthesia in the New Zealand White rabbit. Vet Anaesth Analg 28: 18-25. 